
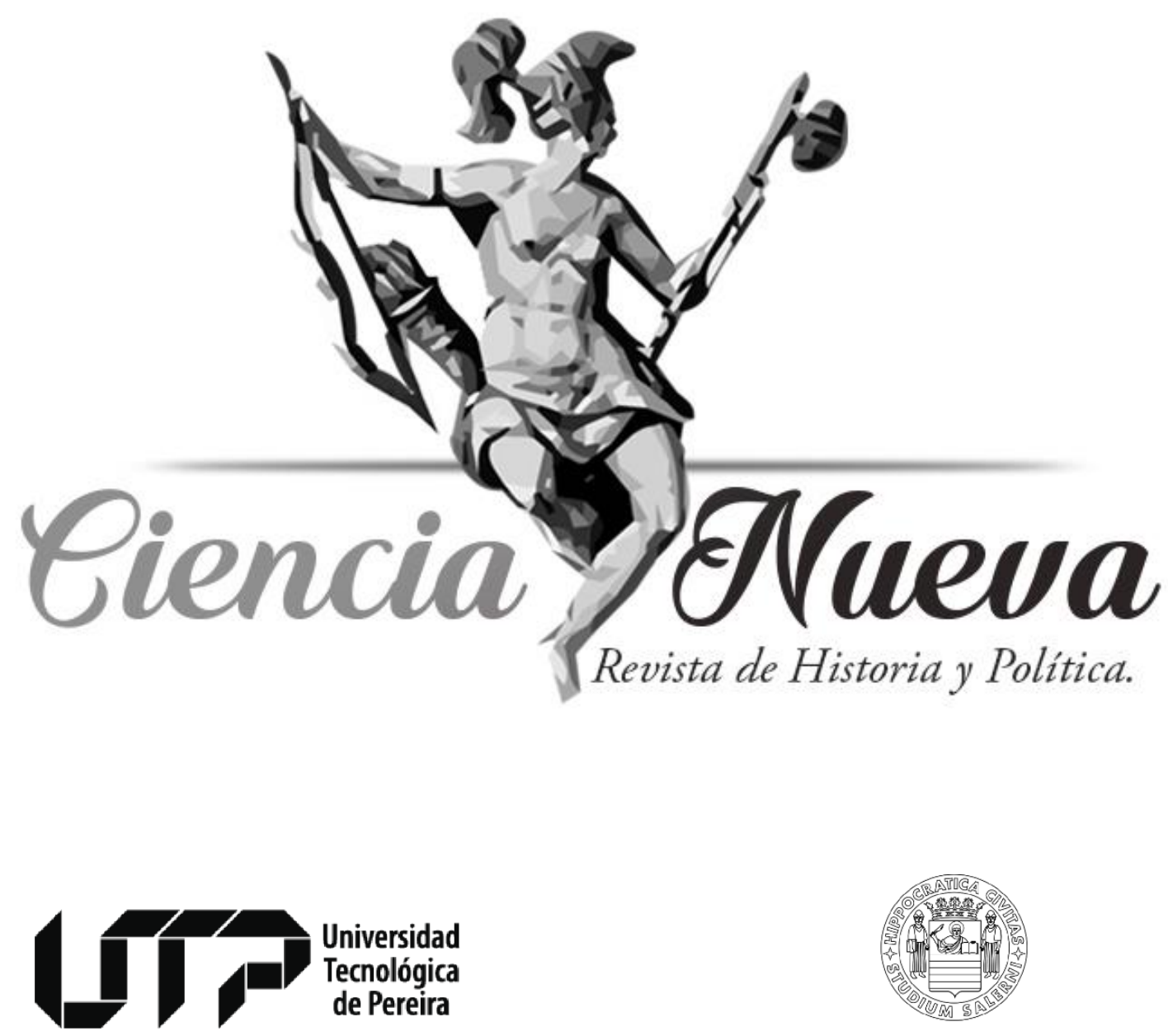

UNIVERSITÀ DEGLI STUDI DI SALERNO

Maestría en Historia

Maestría en Ciencia Política

RESEÑAS

LUIS FELIPE MARÍN GUZMÁN, "LA OTRA CARA DE LA ELECTRIFICACIÓN EN CALDAS: APROXIMACIÓN A LA HISTORIA DE LOS 70 AÑOS DE SINTRACHEC-SINTRAELECOL" (TESIS, UNIVERSIDAD DE CALDAS, FACULTAD DE CIENCIAS JURÍDICAS Y SOCIALES, PROGRAMA DE HISTORIA, 2015), 126 PP.

LUIS FELIPE MARÍN GUZMÁN, "THE OTHER SIDE OF THE ELECTRIFYING IN CALDAS: APPROACH TO THE HISTORY OF THE 70TH ANNIVERSARY OF SINTRACHEC-SINTRAELECOL" (THESIS, UNIVERSIDAD DE CALDAS, FACULTY OF LEGAL AND SOCIAL SCIENCES, UNDERGRADUATE PROGRAM OF HISTORY, 2015), 126 PP.

Freddy Huberto Ríos García pp. 174-176

Vol. $1 N^{o}$ 2, Julio-Diciembre de 2017

Pereira, Colombia 


\section{LUIS FELIPE MARÍN GUZMÁN, "LA OTRA CARA DE LA ELECTRIFICACIÓN EN CALDAS: APROXIMACIÓN A LA HISTORIA DE LOS 70 AÑOS DE SINTRACHEC-SINTRAELECOL" (TESIS, UNIVERSIDAD DE CALDAS, FACULTAD DE CIENCIAS JURÍDICAS Y SOCIALES, PROGRAMA DE HISTORIA, 2015), 126 PP.* \\ LUIS FELIPE MARÍN GUZMÁN, "THE OTHER SIDE OF THE ELECTRIFYING IN CALDAS: APPROACH TO THE HISTORY OF THE 70TH ANNIVERSARY OF SINTRACHEC-SINTRAELECOL" (THESIS, UNIVERSIDAD DE CALDAS, FACULTY OF LEGAL AND SOCIAL SCIENCES, UNDERGRADUATE PROGRAM OF HISTORY, 2015), 126 PP.}

Freddy Huberto Ríos García** freddyhrios@gmail.com

ORCID: http://orcid.org/0000-0002-1683-7302

$\begin{array}{cl}\text { Recibido: } & 24 \text { de abril de } 2017 \\ \text { Revisado: } & 30 \text { de abril de } 2017 \\ \text { Aceptado: } & 09 \text { de junio de } 2017 \\ \text { Publicado: } & 22 \text { de agosto de } 2017\end{array}$

L

uis Felipe Marín estudia el desenvolvimiento a través de la historia del sindicato de la Central Hidroeléctrica de Caldas - CHEC. Da cuenta de las vicisitudes, luchas y conquistas de un grupo sindical sin perder de vista los contextos nacional y mundial que lo influencian, marcan el derrotero y frente a los cuales se desarrolla. Del mismo modo expone minuciosamente las transformaciones del sindicato en sus modos de acción y en su orientación ideológica.

El presente texto, con el cuál el autor opta por el título de historiador, pretende subsanar un vacío historiográfico en torno al movimiento sindical, integrando una mirada que explique "las regiones y localidades desde la perspectiva de los trabajadores".

El autor construye su interpretación apelando a fuentes orales, documentales y bibliográficas. Para las primeras hace uso de las técnicas de entrevista y de grupos focales, mientras que los documentos consultados van desde la prensa hasta los archivos de las oficinas gubernamentales.

Marín Guzmán se propone analizar tres fenómenos temporales que corresponden al mismo número de capítulos que componen su tesis de grado: en el primero capítulo aborda la electrificación como un hito en la modernización de las regiones, comprendiendo las décadas de 1940 a 1970. Para el autor, el propósito nacional de impulsar el esquema de sustitución de importaciones fue un factor determinante para el desarrollo económico regional, tuvo su eco en Caldas con proyectos como el campo de aterrizaje en Santágueda, la fábrica de cementos Caldas y la Central Hidroeléctrica de Caldas. En 1944 se suscribió la escritura que dio nacimiento a la CHEC, que coincidiendo con el centenario de la ciudad,

\footnotetext{
* El presente artículo respeta las directrices y normas dispuestas en la Declaración de Ética de Publicación de Ciencia Nueva, Revista de Historia y Política. Esta declaración puede consultarse en la página web de la revista: revistas.utp.edu.co/index.php/historia

*** Licenciado en Filosofía y candidato a Magíster en Historia por la Universidad Tecnológica de Pereira.
} 
aplazado dos años, tuvo su puesta en servicio en diciembre de 1951. Por su parte, el sindicato de la misma, SINTRACHEC, tuvo su nacimiento en 1946, con el reconocimiento de su personería jurídica por parte del Ministerio de Gobierno. Nacimiento motivado por la pretensión de la empresa de despedir a los obreros no calificados que trabajaron en la construcción de las locaciones y la planta, lo que configuró el primer conflicto laboral en el seno de la CHEC.

Para esta época existía en el ambiente obrero influencias de tipo socialista, de tendencias tanto marxistas como liberales. Lo cual se evidencia en la existencia profusa de periódicos y revistas de germen obrero o sindical donde se preconiza la reivindicación de la clase trabajadora. No obstante fueron los partidos tradicionales quienes cooptaron los diferentes sindicatos y los agruparon ya fuera en la Confederación Sindical de Colombia CSC, que luego cambió su nombre por la Confederación de Trabajadores de Colombia CTC, en el caso del partido liberal; o bajo la Unión de Trabajadores de Colombia - UTC de influencia conservadora.

Precisamente el naciente sindicato de la CHEC, SINTRACHEC, hizo parte en su periodo inicial de la UTC conservadora, al pertenecer a UTRACAL. Esta adscripción le imprimió un tinte de concertación y cooperación a las actuaciones iniciales del sindicato frente a los directivos de la empresa, en las cuales preponderaron las peticiones y requerimientos de corte económico tal como se ven plasmadas en las convenciones.

El segundo capítulo se centra en las décadas que van de 1970 a 1990, y en las disputas y contradicciones políticas que se gestaron en las mismas. La temporalidad estudiada en este capítulo coincide con la aparición de los llamados "cocacolos marxistas", formados en su gran mayoría en el SENA, quienes aportaron a la organización sindical una visión política más amplia, fruto de unos tiempos convulsionados signados por el estatuto de seguridad de $\operatorname{los} 70$ y 80 .

En estos años SINTRACHEC renunció a la UTC e ingresó a la Federación Nacional de Sindicatos de Trabajadores y Empleados Públicos - FENASINTRAP, la cual tenía unos postulados marxista-leninistas. Es en esta época se produjeron los conflictos laborales más agudos y las acciones sindicales más contundentes, como la enmarcada en la huelga de 1982.

Por último, el tercero capítulo aborda la entrada del neoliberalismo al país, desde los 90 hasta el presente. En estas décadas se produce en SINTRACHEC lo que el autor denomina el salto de un sindicato de base a un sindicato de industria, con la inserción de esta dentro del Sindicato de Trabajadores Eléctricos de Colombia - SINTRAELECOL, como respuesta natural del sindicato a la apuesta del Estado por la interconexión del sistema eléctrico nacional, y la asunción de una conciencia de la debilidad de las luchas sindicales atomizadas. En este momento la empresa entró en periodo de capitalización, por lo que se perdió la participación accionaria mayoritaria por parte de los municipios y quedó en manos de las Empresas Públicas de Medellín - EPM. En esta fase el sindicato evolucionó en sus aspiraciones, al pasar de la lucha por las reivindicaciones económicas a la defensa de la empresa como un patrimonio regional. Defensa que no logra conservar la CHEC para los caldenses.

De forma acertada, Marín Guzmán da cuenta del contexto mediante una lectura y puesta en escena de los principales hechos que enmarcan los derroteros mundiales y la vida nacional. Cumple con su cometido, al lograr visibilizar los rostros de los trabajadores, quienes, en palabras del autor, son "la otra cara de la electrificación en el departamento de Caldas". 
Se puede afirmar que el autor logra sortear con éxito su advertencia inicial de no sucumbir al sesgo ideológico a pesar de la militancia declarada por parte del mismo.

Por otro lado el uso de las fuentes es preciso y contundente, por lo que el hilo argumental se entreteje con rigurosidad. Aunque se debe ampliar la citación, sobre todo en referencia a los periódicos consultados.

A pesar de las bondades y aciertos del proceso investigativo, el texto no presenta un análisis suficiente respecto a los últimos años del sindicato. Además, las características y orientaciones del movimiento al interior de SINTRAELECOL, en su subdirección caldense, no logran vislumbrarse de un modo claro.

Queda en el ambiente la sensación de un sindicato fuerte y combativo en su pasado, pero silenciado y anémico en su presente. 